Original Research

\title{
Evaluation of the Psychological Effect of Changes in the Oral Environment
}

\author{
Kikuchi Satomi, DMD \\ Department of Removable Prosthodontics, Tsurumi University School of Dental Medicine \\ (Director: Prof. Hosoi Toshio)
}

\section{Clinical significance}

The Emotion Spectrum Analysis Method (ESAM), which quantifies the time-related emotional state by electroencephalography, was applied to investigate how changes in the oral environment influence the emotional state. The results suggested that we may be able to assess the state of mind as it is influenced by changes in the oral environment.

\section{ABSTRACT}

Purpose : This study investigated the possibility of assessing the emotional state as it is influenced by changes in the oral environment through the use of the Emotion Spectrum Analysis Method (ESAM). Materials and Methods: Comfortable experimental palatal plates (PP) and uncomfortable palatal plates $(\mathrm{D}-\mathrm{PP})$ were worn by 10 subjects with normal dentition. Electroencephalograms (EEG) of five subjects who sensed discomfort $(\mathrm{D}+)$ and of five subjects who did not sense discomfort $(\mathrm{D}-)$ while wearing PP were recorded before and while wearing both types of palatal plates with the subjects' eyes closed according to the International 10-20 Standard. The EEGs were analyzed chronologically using the emotional index based on ESAM, and the analyzed data were compared between the two groups. In addition, the subjects' sensations were evaluated by the Visual Analogue Scale (VAS) and compared between the two groups.

Results : The negative index $(\mathrm{D}-)$ significantly increased while the positive index $(\mathrm{D}+)$ significantly decreased while wearing the palatal plates. The tendency was clearer with $\mathrm{D}-\mathrm{PP}$, which had greater discomfort, and this result corresponded to the sensory evaluation reflected in the VAS score. The sensory evaluation seemed to correspond to the ESAM index that measured the influence of the changes in the oral environment caused by the palatal plates.

Conclusion: The results of this study suggest that it is possible to use ESAM to evaluate the psychological effects caused by changes in the oral environment.

\section{Key words}

electroencephalogram, emotion, emotional index, experimental palatal plate, emotion spectrum analysis method (ESAM)

\footnotetext{
Corresponding author: Kikuchi Satomi

2-1-3, Tsurumi, Tsurumi-ku, Yokohama, Kanagawa 230-8501, Japan

Tel : +81-45-581-1001, Fax : +81-45-573-9599

E-mail : kikuchi-satomi @ tsurumi-u.ac.jp

Received on March 31, 2005/Accepted on August 8, 2005
} 


\section{INTRODUCTION}

Evaluation of dental treatment currently tends to depend on patients' subjectivity. The methods of evaluating a patient's psychological state according to physiological indexes are not often applied in the field of dentistry. Quantifying time-related psychological effects caused by changes in the oral environment may provide a new method of evaluating prosthetic treatment. Recent studies suggest the usefulness of electroencephalography (EEG) as a physiological index for evaluation of prosthetic treatment ${ }^{1-5)}$. Musha et al. established the Emotion Spectrum Analysis Method (ESAM) ${ }^{6,7)}$, which chronologically quantifies the psychological state with EEG. The purpose of this study was to establish a new psychological evaluation method of prosthetic treatment. ESAM was investigated as a tool for evaluating the psychological effect of changes in the oral environment caused by palatal plates.

\section{MATERIALS AND METHODS}

\section{Subjects}

Ten subjects ( 5 males and 5 females; mean age of $23.8 \pm 1.0$ years) with normal occlusion were selected from the students at Tsurumi University School of Dental Medicine. None of them reported any subjective or objective abnormalities in their stomatognathic function systems. They had no medical history of any diseases that would influence the EEG. After the subjects were informed about the purpose and the details of this study, they agreed to cooperate. This study was carried out with the approval of Tsurumi University ethics review committee.

\section{Palatal Plates and Subject Groups}

1. Fabrication of experimental palatal plates After impressions of the subjects' maxilla were made with alginate impression material (AROMA FINE DF III, GC, Tokyo, Japan), working casts were fabricated with dental plaster (NEW ZO-STONE, SHIMOMURA GYPSUM, Saitama, Japan). The margins of the palatal plate were on the cervical line of the palatal side of the teeth and on the vibrating line. Self-curing tray material (OSTRON II, GC) was uniformly adapted to the casts to make $2.0 \mathrm{~mm}$ thick resin plates (experimental palatal plates ; PP) (Fig. 1). To increase the subjects' discomfort, another type of palatal plate with $0.5 \mathrm{~mm}$ high projections on the mucosal surface at the incisive papilla (uncomfortable palatal plates; $\mathrm{D}-\mathrm{PP}$ ) was made. Both types of palatal plates were polished, examined with white silicone material (FIT CHECKER, GC) to confirm the adaptation to the mucosa, and trimmed so that they did not interfere with the occlusion.

2. The subjects were divided into two groups Those who subjectively felt strong discomfort when wearing $\mathrm{PP}(\mathrm{D}+)(\mathrm{n}=5)$ and those who did not feel such discomfort $(D-) \quad(n=5)$.

\section{Sensory Evaluation Using the Visual Analogue Scale (VAS)}

The subjects' sensory evaluations when wearing $\mathrm{PP}$ and $\mathrm{D}-\mathrm{PP}$ were performed using the Visual Analogue Scale (VAS) ${ }^{8)}$ with every EEG measurement. To measure the discomfort caused by the $\mathrm{PP}$ and $\mathrm{D}-\mathrm{PP}$, the subjects made a

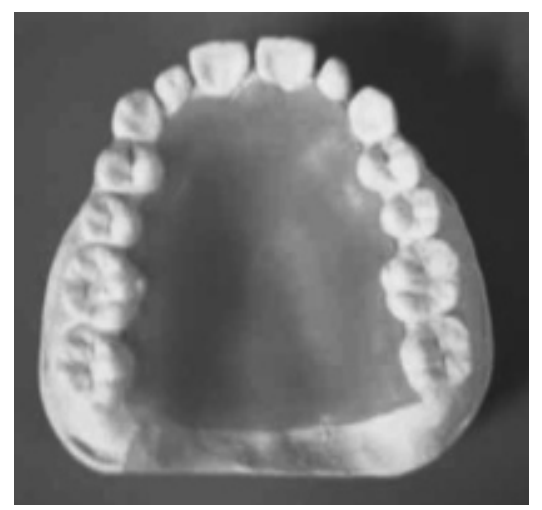

Fig. 1 Experimental palatal plate 


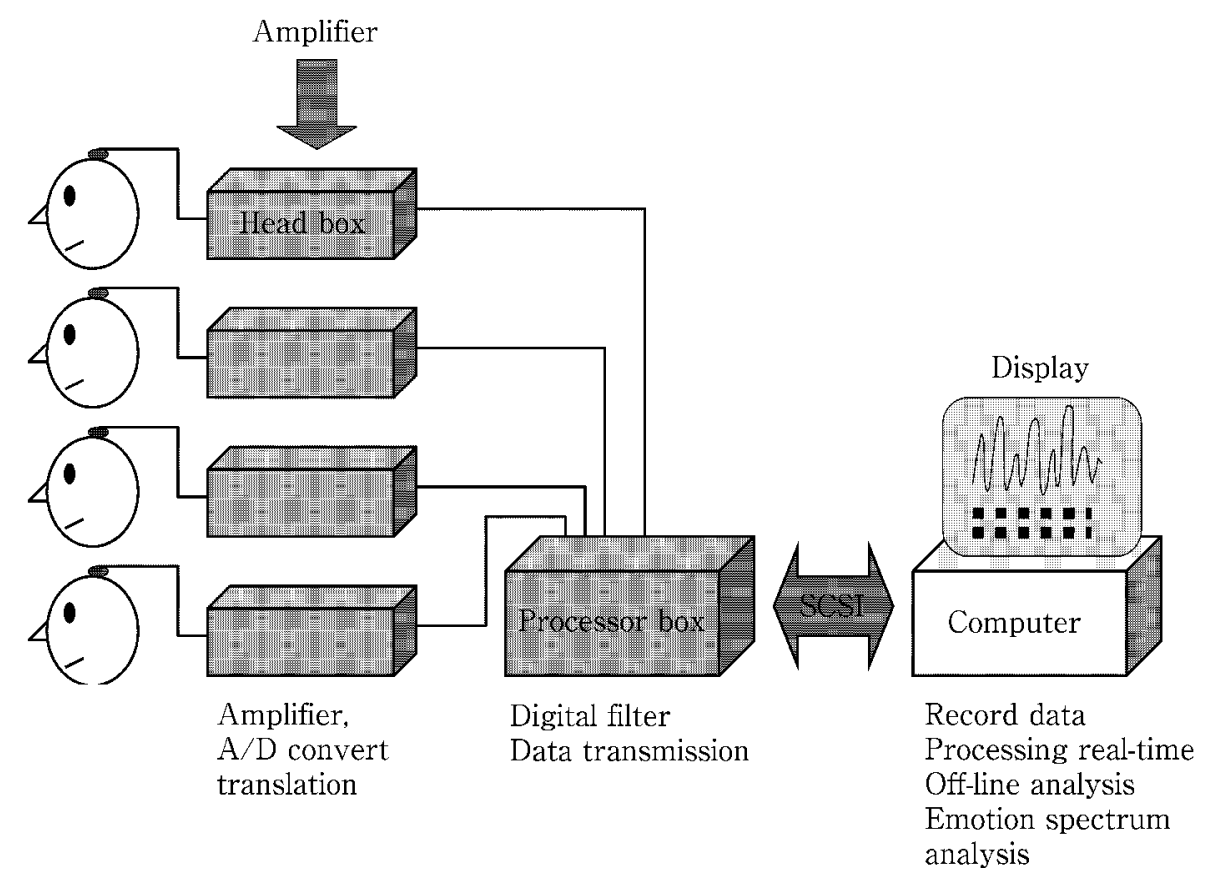

Fig. 2 Diagram of the emotion spectrum analysis system

checkmark on a $100 \mathrm{~mm}$ long horizontal line on which the extreme left (0) indicated no discomfort and the extreme right (100) indicated the highest level of discomfort. The length from the extreme left to the check mark was recorded as the discomfort score.

\section{Method of Recording the EEGs}

The EEGs were recorded in a conference room (approximately $30 \mathrm{~m}^{2}$ ) at Tsurumi University Dental Hospital. Noise and artifacts were carefully avoided during the EEG recording, and the room temperature was kept at about 21 to $24^{\circ} \mathrm{C}$.

To record the EEGs, the Emotion Spectrum Analyzing System (Brain Functions Laboratory, Inc., Kanagawa, Japan) was used (Fig. 2). The unipolar derivation method with a reference electrode on the right earlobe was used to record the EEG. Ten silver/silver-chloride disk electrodes (6 mm dia.) were placed on the scalp according to the International 10-20 standard ${ }^{9}$ (Fig. 3) with electroconductive adhesive. The extracted EEG data were recorded at every $10 \mathrm{~ms}$, and the emo-

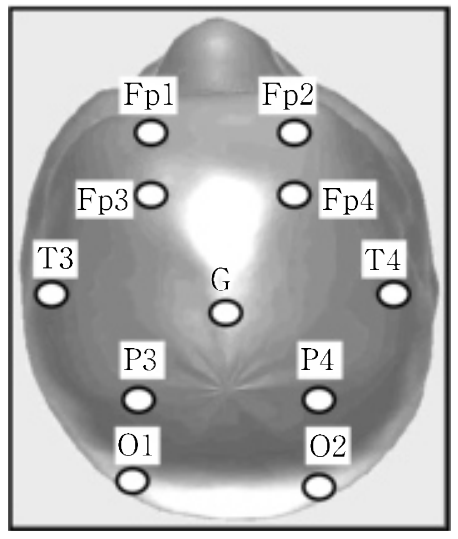

Fig. 3 Electrode layout of the International $10^{-}$ 20 Standard

tion analysis was performed with analysis software (Emotion Expert, Brain Functions Laboratory, Inc.) every 5.12 seconds.

\section{Emotion Spectrum Analysis Method (ESAM)}

In ESAM, there are 45 electrode pairs and 135 cross-correlation coefficients (forming a 135dimensional vector, or $135^{-}$vector) calculated in the theta, alpha and beta frequency bands in every 5.12 seconds, from which the four orthogonal emotional states [anger/stress (N 1), joy/ satisfaction (P 1), sadness/depression (N 2) and relaxation $(\mathrm{R})]$ were separated and their levels 


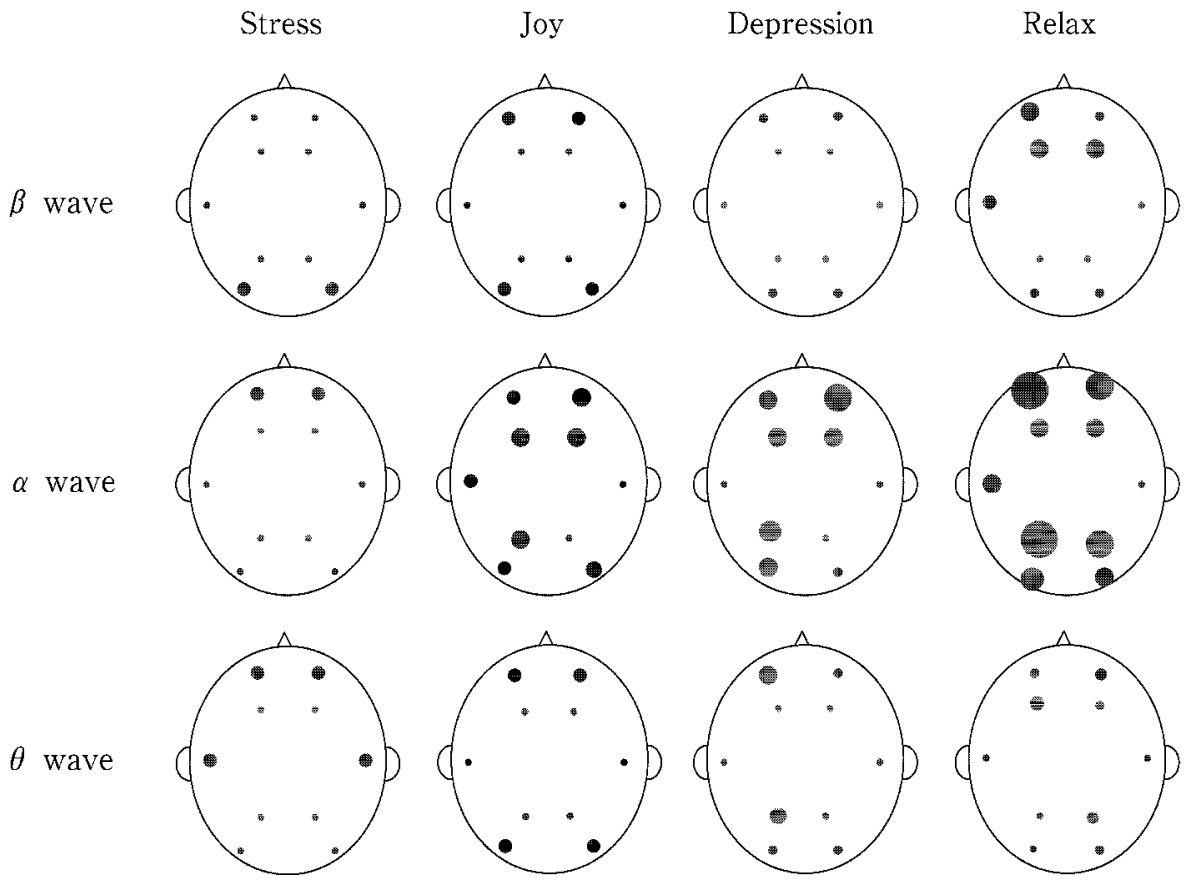

Fig. 4 Contribution of brain wave data for each emotional index of the standard emotion matrix (SM)

evaluated by means of linear transformation. The transformation was performed by operating the standard emotion matrix (SM), on the 135vector. General emotional states are expressed by superimposing four basic emotional states ( $\mathrm{N}$ 1, N 2, P 1, and R). In addition, to simplify the situation, we derived a customized emotion matrix $(\mathrm{CM})$, based on mental stress $(\mathrm{N})$ and joy $(\mathrm{P})$, as a discomfort index on the same scale on which $\mathrm{P}$ is the starting point (0) expressing the least discomfort, and $\mathrm{N}$ is the terminal point (100) expressing extreme discomfort.

Figure 4 shows the contribution of the brain wave data to each emotional factor. The information on the phase and location relationship between electrodes at the scalp potential level of each emotional state ( N 1, N 2, P 1, and R) was calculated over time as cross-correlation coefficients of the potentials between each electrode. A database was then made ${ }^{6,7)}$ by analyzing the EEGs of 7 subjects who were well-trained at imagining anger, joy, sadness and relaxation. The emotion spectrum analyzing software (Emo- tion Expert) was originally equipped with this database as the universal emotion database.

The calculation of the standard emotion matrix (SM) from the EEG at certain emotional states was performed as follows:

The potential collected with electrode $\mathrm{j}$ as a function of time $t$, then the cross-correlation coefficient $X_{j \mathrm{k}}$ of the potentials collected with electrodes ${ }_{j}$ and ${ }_{k}$ are defined as

$$
\begin{aligned}
& \mathrm{X}_{\mathrm{jk}}=\left\langle\mathrm{X}_{\mathrm{j}}(\mathrm{t}) \mathrm{X}_{\mathrm{k}}(\mathrm{t})\right\rangle /\left\langle\mathrm{X}_{\mathrm{j}}{ }_{\mathrm{j}}(\mathrm{t})\right\rangle 0.5\left\langle\mathrm{X}_{\mathrm{k}}{ }_{\mathrm{k}}(\mathrm{t})\right\rangle^{0.5} \\
& (\langle\rangle \text { is the ensemble average })
\end{aligned}
$$

The number of pairs out of ten electrodes is ${ }_{10} \mathrm{C}^{2}=45$. The EEG measurement bands are narrowed down to 5 to $20 \mathrm{~Hz}$. They were divided into 3 frequency bands of 5 to $8 \mathrm{~Hz}(\theta$ wave), 8 to $13 \mathrm{~Hz}$ ( $\alpha$ wave), and 13 to $20 \mathrm{~Hz}$ ( $\beta$ wave) frequency bands. The cross-correlation coefficients of the formula above were calculated for each frequency band. This process gives us $45 \times 3=135$ cross-correlation coefficients since there are 45 variables in each frequency band. Since the combination of these variables can be regarded as vectors, the 135 cross-correlation 
coefficients are called the " 135 dimensional input vectors," which are multiplied by the universal emotional database mentioned above. Thus, the standard emotion matrix (SM) is obtained.

\section{Experimental Tasks and Analyses of EEGs}

In the preliminary experiment, it was confirmed whether or not the palatal plates (PP) influenced the EEG. The control was defined as the condition before the palatal plates were applied, and it was verified that ESAM could record the change in each emotional index while the subjects wore the palatal plates. The rate of change of each emotional index of the standard emotion matrix (SM) compared to the control was evaluated between the $\mathrm{D}+$ group (subjects who sensed discomfort with PP) and the Dgroup (the remaining five subjects who did not sense discomfort with $\mathrm{PP}$ ).

In the main experiment, $\mathrm{PP}$ and $\mathrm{D}-\mathrm{PP}$ were applied and the rate of change of the emotional indexes of the standard emotion matrix (SM) for both types of plates compared to the control was evaluated between the $\mathrm{D}+$ group and the $\mathrm{D}-$ group. In addition, the same comparison was made for the discomfort index of the customized emotion matrix (CM) while the subjects wore PP and $\mathrm{D}-\mathrm{PP}$

1. 1-Preliminary experiment

The subjects were instructed to be seated and to keep their eyes closed while recording the EEGs, except for placement and removal of the palatal plate. The EEG of each subject was recorded with $\mathrm{PP}$ once a day according to the following procedures.

The subjects were instructed to rest for 5 minutes with their eyes closed after the setup, and the measurement was carried out in the following sequence after it was confirmed that there were no artifacts in the EEGs:

1) 2 minutes' rest with eyes closed (control)

2) placement of $\mathrm{PP}$
3) 1 minute rest with eyes closed after confirming that there were no artifacts

The rates of change to the control in each emotional index of the standard emotion matrix (SM) for PP was calculated by ESAM for D+ and $\mathrm{D}-$. It was verified that ESAM could detect changes in the oral environment caused by PP. The data were compared between the two groups using the Mann-Whitney U test.

\section{2-Main experiment}

The subjects were instructed to rest and keep their eyes closed while the EEG was recorded except for the placement and removal of the palatal plate and recording of the VAS. The type of palatal plate was chosen randomly ; the subjects were not informed beforehand which type of palatal plate would be used. The EEGs were taken on the same day once per day for each type of palatal plate using the following procedures for both $\mathrm{PP}$ and $\mathrm{D}-\mathrm{PP}$.

The subjects were instructed to rest for 5 minutes with their eyes closed after the setup, and the measurements were made according to the following sequence after it was confirmed that there were no artifacts in the EEGs.

1) 2 minutes' rest with eyes closed (control)

2) Placement of $\mathrm{PP} / \mathrm{D}-\mathrm{PP}$

3) 1 minute rest with eyes closed after confirming of no artifact

4) VAS was recorded

5) removal of $\mathrm{PP} / \mathrm{D}-\mathrm{PP}$

6) 3 minutes' rest

The experimental sequence above was repeated twice a day for both $\mathrm{PP}$ and $\mathrm{D}-\mathrm{PP}$, and it was performed 5 times for each type of palatal plate for a total of 10 times for each subject.

The rates of change compared to the control (the EEG data of the second 1 minute were used) for each emotional index of the standard emotion matrix (SM) with PP and D-PP were calculated by ESAM as $\mathrm{D}+$ and $\mathrm{D}-$. The data were compared between the two groups with the 
Mann-Whitney U test, which was also used to statistically analyze the discomfort index of the customized emotion matrix (CM). All statistical analyses were performed using SPSS $12.0 \mathrm{~J}$ for Windows (SPSS Japan Inc., Tokyo, Japan).

\section{RESULTS}

\section{Preliminary Experiment}

Changes in the each emotional index of the standard emotion matrix (SM) while PP was worn compared between $\mathrm{D}+$ and $\mathrm{D}-$ (Fig. 5).

The rate of change of $\mathrm{N} 1$ for $\mathrm{D}+$ significant ly increased $(\mathrm{p}<0.05)$ for the palatal plates, while $\mathrm{P} 1$ and $\mathrm{R}$ significantly decreased compared to $\mathrm{D}-$. Another negative index (N 2) tended to increase $\mathrm{D}+$ in comparison to $\mathrm{D}-$, but there was no significant difference.

\section{Main Experiment}

1. Comparison of the rates of change for each emotional index of the standard emotion matrix (SM)

The rate of change of each emotional index of the standard emotion matrix (SM) while wearing $\mathrm{PP}$ and $\mathrm{D}-\mathrm{PP}$ compared to the control was evaluated for $\mathrm{D}+$ and $\mathrm{D}-$ (Figs. 6 and 7).

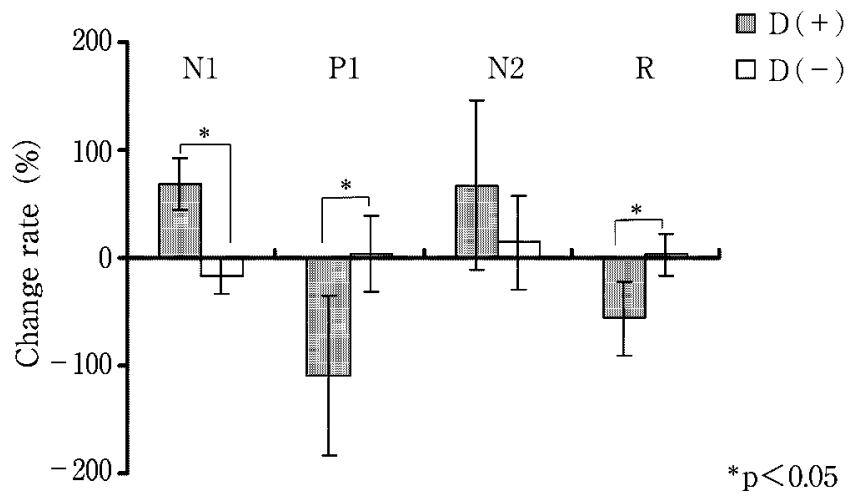

Fig. 5 Results of the preliminary experiment Rate of change of each emotional index of the standard emotion matrix (SM) while PP was worn between $\mathrm{D}+$ and $\mathrm{D}-$ compared to the control. There are significant differences between $\mathrm{D}+$ and $\mathrm{D}-$ for $\mathrm{N} 1, \mathrm{P} 1$, and $\mathrm{R}$
There were significant differences $(\mathrm{p}<0.05)$ in $\mathrm{N} 1, \mathrm{P} 1$, and $\mathrm{R}$ between $\mathrm{D}+$ and $\mathrm{D}-$ while PP was worn. While D-PP was worn, there was a significant difference $(\mathrm{p}<0.05)$ only in $\mathrm{N} 1$ between $\mathrm{D}+$ and $\mathrm{D}-$.

A comparison of the rates of change for each emotional index of the standard emotion matrix (SM) for $\mathrm{PP}$ and $\mathrm{D}-\mathrm{PP}$ in $\mathrm{D}+$ and $\mathrm{D}-$ is shown in Table 1. There were significant differences $(\mathrm{p}<0.05)$ in $\mathrm{N} 1, \mathrm{P} 1$, and $\mathrm{R}$ of the standard emotion matrix $(\mathrm{SM})$ for $\mathrm{D}+$.

For $\mathrm{D}-$, there was no significant difference

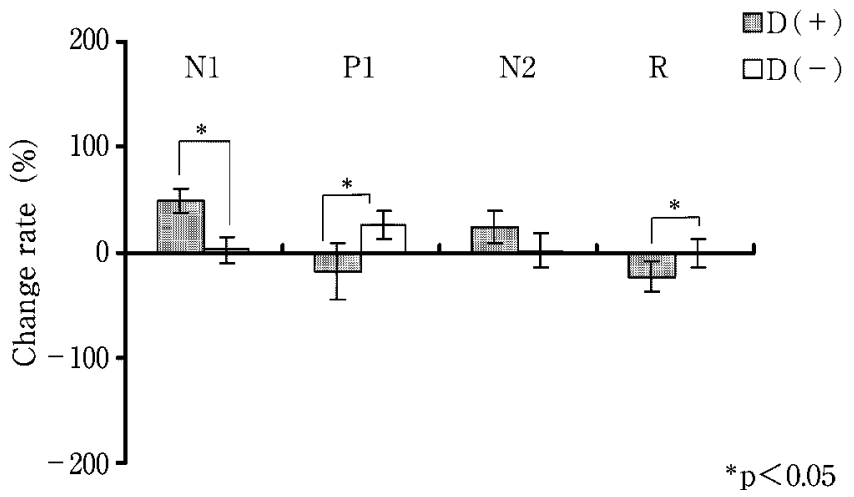

Fig. 6 Results of the main experiment

Rates of change of each emotional index of the standard emotion matrix (SM) while PP was worn between $\mathrm{D}+$ and $\mathrm{D}-$ compared to the control. There are significant differences between $\mathrm{D}+$ and $\mathrm{D}-$ for $\mathrm{N} 1, \mathrm{P} 1$, and $\mathrm{R}$

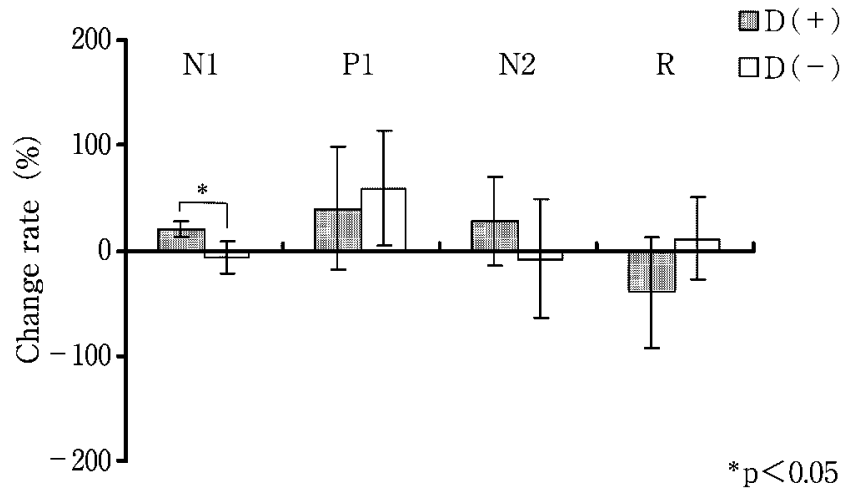

Fig. 7 Results of the main experiment

Rates of change of each emotional index of the standard emotion matrix (SM) while wearing $\mathrm{D}-\mathrm{PP}$ between $\mathrm{D}+$ and $\mathrm{D}-$ compared to the control. There is a significant difference between $\mathrm{D}+$ and $\mathrm{D}-$ in $\mathrm{N} 1$ 
Table 1 Rates of change of each emotional index of the standard emotion matrix (SM) between $\mathrm{PP}$ and $\mathrm{D}-\mathrm{PP}$ in $\mathrm{D}+$ and $\mathrm{D}-$ There are significant differences in $\mathrm{N} 1$, $\mathrm{P} 1$, and $\mathrm{R}$ in $\mathrm{D}+$. There is no significant difference in any of the emotional indexes in $\mathrm{D}-$

\begin{tabular}{ccc}
\hline \hline SM & $\mathrm{D}(+)$ & $\mathrm{D}(-)$ \\
\hline $\mathrm{N} 1$ & $*$ & \\
$\mathrm{P} 1$ & $*$ & \\
$\mathrm{~N} 2$ & & \\
$\mathrm{R}$ & $*$ & \\
\hline${ }^{*} \mathrm{p}<0.05$ & &
\end{tabular}

between $\mathrm{PP}$ and $\mathrm{D}-\mathrm{PP}$ in the rates of change of any emotional index of the standard emotion matrix (SM).

2. Sensory evaluation by the Visual Analogue Scale (VAS)

Figure 8 shows the results of the sensory evaluation using the VAS while PP and D-PP were worn for $\mathrm{D}+$ and $\mathrm{D}-$. The application of either PP or D-PP caused the higher level of discomfort in $\mathrm{D}+$ compared to $\mathrm{D}-$ with a significant difference $(p<0.05)$. The application of $\mathrm{D}-\mathrm{PP}$ caused a significantly higher level $(\mathrm{p}<$ 0.05 ) of discomfort in $\mathrm{D}+$, but there was no significant difference between $\mathrm{PP}$ and $\mathrm{D}-\mathrm{PP}$ in $\mathrm{D}-$.

3. Comparison of the discomfort index of the customized emotion matrix (CM)

Figure 9 shows the results of the comparison between $\mathrm{D}+$ and $\mathrm{D}-$ for the discomfort index of the customized emotion matrix $(\mathrm{CM})$ while PP and $\mathrm{D}-\mathrm{PP}$ were worn. Application of $\mathrm{PP}$ and $\mathrm{D}-\mathrm{PP}$ caused significantly higher level $(\mathrm{p}<$ $0.05)$ of discomfort in $\mathrm{D}+$ compared to $\mathrm{D}-$. The application of D-PP caused a significantly higher level $(\mathrm{p}<0.05)$ of discomfort in $\mathrm{D}+$, but there was no significant difference between PP and $\mathrm{D}-\mathrm{PP}$ in $\mathrm{D}-$.

\section{DISCUSSION}

It is important in the field of dentistry to objectively evaluate the effect of prosthetic treat-

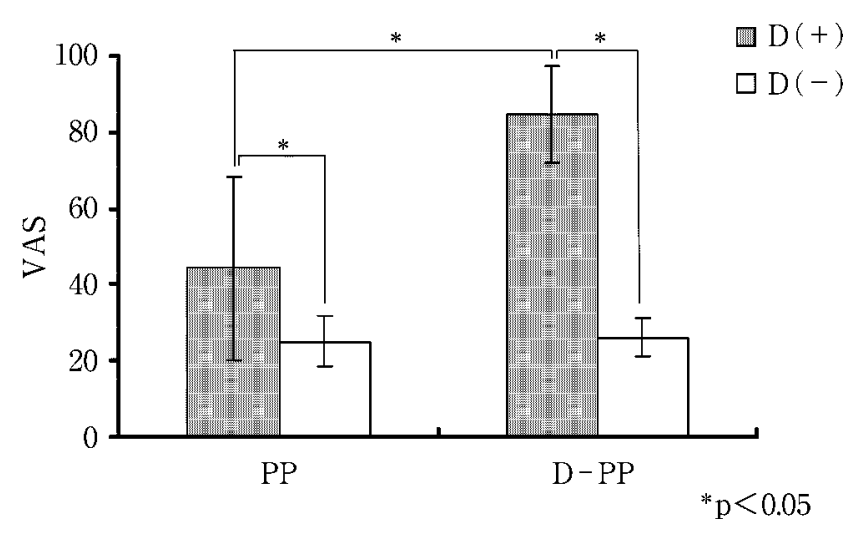

Fig. 8 Comparison of the sensory evaluation using the Visual Analogue Scale (VAS) while PP and $\mathrm{D}^{-}$ $\mathrm{PP}$ were worn between $\mathrm{D}+$ and $\mathrm{D}-$. " 0 " indicates the least discomfort and " 100 " indicates the greatest discomfort

There are significant differences between $\mathrm{D}+$ and $\mathrm{D}-$ for both $\mathrm{PP}$ and $\mathrm{D}-\mathrm{PP}$. There is a significant difference between $\mathrm{PP}$ and $\mathrm{D}-\mathrm{PP}$ in $\mathrm{D}+$

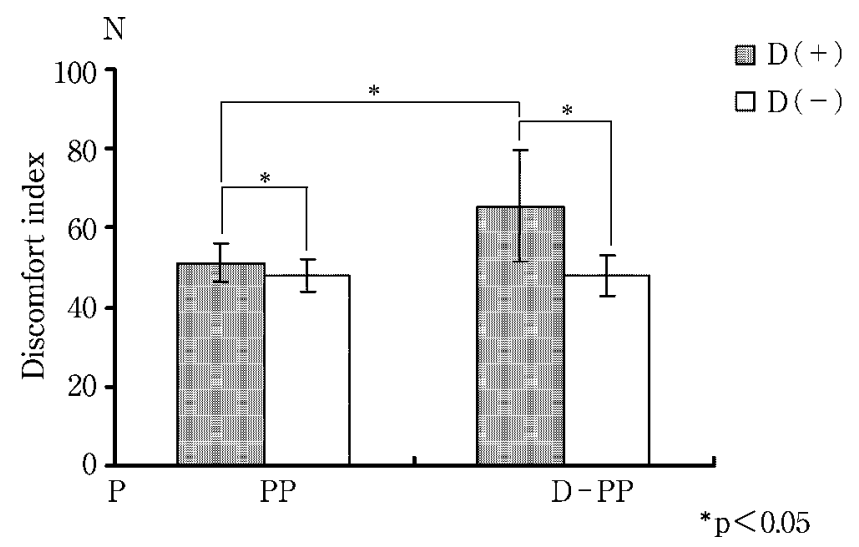

Fig. 9 Results of the comparison of the discomfort index of the customized emotion matrix (CM) between $\mathrm{D}+$ and $\mathrm{D}-$ while $\mathrm{PP}$ and $\mathrm{D}-\mathrm{PP}$ were worn. N (100) indicates the greatest discomfort and $\mathrm{P}(0)$ indicates the least discomfort There are significant differences between $\mathrm{D}+$ and $\mathrm{D}-$ in both PP and D-PP. There is a significant difference between $\mathrm{PP}$ and $\mathrm{D}-\mathrm{PP}$ in $\mathrm{D}+$

ment. The analysis of mandibular function ${ }^{10,11)}$ has been used as a method of evaluating prosthetic treatment. Recent studies suggest the usefulness of EEG as a physiological measure ${ }^{1-5}$. This study investigated the use of $\mathrm{ESAM}^{6,7)}$, which quantifies time-related psychological states based on EEGs, and the psychological effect of changes in the oral environment caused 
by palatal plates.

Mushimoto et al. ${ }^{4}$ and Ichikawa ${ }^{5)}$ used EEGs in the field of dentistry and reported that the percent power of the alpha wave $(\% \alpha)$ reflected the change of emotion caused by oral sensation, and it was possible to detect the subject's difficulties with masticatory function. Yamamura et al. $^{2}$ ) analyzed the percent of changes in EEGs, resulting from the application of a palatal plate, and mentioned the usefulness of EEGs in prosthetic dentistry. These analyses divided EEG into 4 frequency bands, which were calculated as a percentage of the total power of 4 frequency bands. However, these data did not provide clinically significant data.

Nishiyama et al. ${ }^{12)}$ used ESAM to clinically evaluate psychological states with EEG. The relationship between the psychological changes before and after mastication as analyzed by ESAM and the effect of time-related factors of masticatory rhythm caused by an occlusal interference was investigated. As a result, the occlusal interference caused sensory change of the oral cavity, which affected masticatory rhythm ; stress (N 1) tended to increase while relaxation (R) tended to decrease in all subjects. This result suggested that a negative change in oral sensation caused by the occlusal interference led to an increase in the negative emotional state and a decrease in the emotional state. It was also suggested that the capability of detecting negative changes in oral sensation was higher in $\mathrm{N} 1$ as a negative index, and $\mathrm{R}$ as a positive index. The present study was carried out based on these results. The reasons for introducing ESAM into prosthetic dentistry are that it can measure psychological states in clinically intelligible emotional factors and that it might be possible to measure EEGs in motion. The appropriateness and the reliability of assessing psychological states using ESAM have been verified by Musha, and ESAM has been applied to various types of study (for example, a study on a method of evaluating software usability with $\mathrm{EEG}^{13)}$ ).

The methods of this study were carefully considered in order to produce reliable results. Since age influences $\mathrm{EEG}^{9)}, 10$ subjects of mean age \pm 1.0 year were selected to avoid age differences among the subjects. The subjects were divided into two groups : five subjects who subjectively felt discomfort with PP $(\mathrm{D}+)$ and five subjects who did not feel discomfort $(\mathrm{D}-$ ), in order to verify the possibility of measuring sensory differences among subjects. Thus, it was verified if the sensory differences corresponded to the assessment under the same condition using ESAM as in the preliminary experiment.

$\mathrm{PP}$ and $\mathrm{D}-\mathrm{PP}$ were carefully adjusted not to cause the vomiting reflex, occlusal interference, or pain ${ }^{14,15)}$. In other words, the noise caused by muscle activity was reduced as much as possible. $\mathrm{D}-\mathrm{PP}$, with its $0.5 \mathrm{~mm}$ high projection on the mucosal surface at the incisive papilla, was expected to cause discomfort to the subjects. Considering a study that reported that the pain threshold of the incisive papilla was lower than that of the posterior area of the palate, or central sides of the palate ${ }^{16)}$, it was expected that the projection on the mucosal surface would cause more discomfort than PP. All of the subjects in the $\mathrm{D}+$ group reported discomfort and difficulty.

The VAS is often used as a measurement of patient satisfaction in prosthetic treatment ${ }^{17}$. Kawai et al. verified its reliability and validity ${ }^{8}$. The VAS was used for subjective evaluation of psychological effect caused by the change in the oral environment from the palatal plate. In this study, it was expected that the subjective VAS results would correspond to the objective ESAM results. A correlation was found between these evaluations as follows.

EEGs change considerably, even in normal subjects, due to factors such as blinking and changes in mental condition ${ }^{9}$. Therefore, the 
subjects were instructed to keep their eyes closed during all EEG measurements to avoid artifacts, and also to avoid the influence of external sensory stimulation ${ }^{18-20)}$. The mental condition of the subjects was also considered. Extraneous noise and the measurements themselves were carefully controlled so as not to influence the subjects' concentration or mental activity. However, the possibility that the experiment itself might have made subjects nervous or excited cannot be denied. In addition, repetition of the experiment gave the subjects experience, which might have influenced the EEG.

To prevent fatigue caused by the repetition of the experiment, the measurements were repeated no more than 2 times, and sufficient rest was provided between the measurements so that the after-effects of the first experiment would not remain in the second experiment. The subjects were instructed to rest for 5 minutes after the set-up to calm any anxiety about changes in the control condition.

Fig. 5 shows the rate of change of the negative emotional indexes, $\mathrm{N} 1$, in the preliminary experiment, which indicated a marked increase, while the positive emotional indexes, $\mathrm{P} 1$ and $\mathrm{R}$, decreased in $\mathrm{D}+$. Obvious changes or inclination did not appear in $\mathrm{D}-$, and this result suggested that the influence of PP was as small as the psychological effect. These results suggested that the subjectivity of the subjects corresponded to the emotional index of $\mathrm{N} 1$ of the standard emotion matrix (SM) of ESAM.

In the main experiment, the results of the comparison between $\mathrm{D}+$ and $\mathrm{D}-$ on the rates of change of each emotional index of the standard emotion matrix (SM) while wearing PP were the similar to the preliminary experiment. These findings suggested that the deflection each day and the difficulty of the reproduction of the EEG measurement could be reduced by expressing the results of the experiment under the same condi- tions in rates of change. While wearing $\mathrm{D}-\mathrm{PP}$, there was a significant difference only in $\mathrm{N} 1$ among the subjects or between the two groups, but there was no significant difference in N 2, P 1, or $\mathrm{R}$. The reason there was not much difference in the emotional indexes of the standard emotion matrix (SM) between the two groups needs to be investigated with more subjects. The reasons the stress level (N 1) did not increase with the increase of the task by applying $\mathrm{D}-\mathrm{PP}$ is perhaps because the stress level was already high due to experiencing the task, and/or the subjects' not knowing which type of palatal plate was going to be used caused the increase in stress.

The result of the comparison of the rate of change for each emotional index of the standard emotion matrix (SM) with $\mathrm{PP}$ and $\mathrm{D}-\mathrm{PP}$ indicated the occurrence of the stronger discomfort with $\mathrm{D}-\mathrm{PP}$ than $\mathrm{PP}$ in $\mathrm{D}+$. There was no significant difference in $\mathrm{D}-$ between each emotional index of the standard emotion matrix (SM) while wearing $\mathrm{PP}$ and $\mathrm{D}-\mathrm{PP}$. The results of the standard emotion matrix (SM) seemed to correspond to the results of the VAS. Furthermore, the results of the comparison of each emotional index of the customized emotion matrix $(\mathrm{CM})$ between $\mathrm{D}+$ and $\mathrm{D}-$ while wearing $\mathrm{PP}$ and $\mathrm{D}-\mathrm{PP}$ also corresponded to the VAS results. These results suggested that the psychological assessment using the emotional indexes of ESAM was appropriate. The discomfort index may be more suitable for applying ESAM to prosthetic dentistry because it can make assessment using a relatively subjective scale.

\section{CONCLUSION}

In this study, the Emotion Spectrum Analysis Method (ESAM), which quantifies time-related psychological states by EEG, was applied, and the psychological effect of changes in the oral environment caused by wearing a palatal plate 
was investigated. The results suggested that it may be possible to evaluate the psychological changes caused by changes in the oral environment by using emotional indexes based on ESAM. The systematic combination of ESAM and mandibular function analysis may offer a new vision of an evaluation method in clinical dentistry.

Acknowledgements : The author greatly appreciates Professor Hosoi Toshio, Director of Department of Removable Prosthodontics, Tsurumi University School of Dental Medicine, for his helpful advice on this paper. Mr. Musha Toshimitsu, the president of Brain Functions Laboratory, Inc., is also appreciated for his valuable advice. The author also wishes to thank Dr. Onuki Masari, Assistant Professor, and Dr. Nishiyama Yuichiro, who always gave valuable advice. All the staff of the Department of Removable Prosthodontics are much appreciated, and the volunteers are also appreciated for their participation in this study.

This research was supported by the Academic Frontier Project for Private Universities : matching fund subsidy from the Ministry of Education, Culture, Sports, Science and Technology (2003-2007). A part of this study was presented at the $111^{\text {th }}$ Scientific Meeting of Japan Prosthodontic Society (May 22, 2004, Tokyo).

\section{REFERENCES}

1) Seki M. Influence of Experimental occlusal interference on stomatognathic system - especially concerns with EEG findings-. J Jpn Prosthodont Soc 36: 968-979, 1992. (in Japanese)

2) Yamamura O, Ohhashi K, Maeda $K$ et al. The prosthetic application of the clinical electrocephalograph (SYNAFIT 2100)-Part 1 the way of the experiment. J Jpn Prosthodont Soc 40 : 1044-1049, 1996. (in Japanese)

3) Yamamura O, Maeda K, Ohhashi K et al. The prosthetic application of the clinical electrocephalograph (SYNAFIT 2100) - Part 3 the effects by bite rising. J Jpn Prosthodont Soc 43 : 495-498, 1999. (in Japanese)

4) Mushimoto E, Tanaka H, Ichikawa D. The effect of a change of oral environment accordance with an experimental palatal plate and occlusal interference on electroencephalograms. J Jpn Prosthodont Soc $45: 295-304,2001$. (in Japanese)

5) Ichikawa D. The effect of experimental occlusal interference on electroencephalograms by comparing psychophysiological response and masticatory muscle activity. J Jpn Prosthodont Soc 45:305-314, 2001. (in Japanese)

6) Musha $T$, Terasaki $Y$, Takahashi $T$ et al. Numerical estimation of the state of mind. Methodologies for the conception, design, and application of intelligent systems proceedings of IIZUKA : 25-29, 1996.

7) Musha T, Terasaki Y, Hasnine A et al. Feature extraction from EEGs associated with emotions. Art Life Robotics 1:15-19, 1997.

8) Kawai Y, Machida T, Gunji A et al. Reliability and validity of the Japanese version of the visual analogue scale as an outcome measurement of complete denture prostheses. Prosthodont Res Pract 2:64-71, 2003.

9) Okuma T. Clinical electroencephalography, 4th ed. 81-90, Tokyo : Igaku-Shoin Ltd., 1991. (in Japanese)

10) Hirai T, Matai $N$, Anzai $T$ et al. Masticatory rhyth$\mathrm{m}$ of complete denture wearers analysis by functional evaluation method. J Jpn Prosthodont Soc $30: 12-19,1986$. (in Japanese)

11) Araki T. A study on occlusal adjustment and its estimation method of curative effect in complete denture wearers. J Jpn Prosthodont Soc 45:93-105, 2001. (in Japanese)

12) Nishiyama Y, Kikuchi S, Kobayashi M et al. Study on the evaluation of psychological effect of prosthetic tTreatment. $82^{\text {nd }}$ General Session \& Exhibition of the International Association for Dental Research : 75 (0424), 2004.

13) Tagaito H. An Evaluation of user interface with emotions. UNISYS TECHNOLOGY REVIEW 64: 103-114, 2000. (in Japanese)

14) Furuya M, Yoshinaka M, Ono $T$ et al. Influence of an experimental palatal plate on discriminative ability for thickness. J Jpn Prosthodont Soc 47 : 20-27. 2003. (in Japanese)

15) Furuya M, Yoshinaka M, Ikebe $K$ et al. Influence of thickness and material of experimental palatal plate on gustatory response. J Jpn Prosthodont Soc 48: 67-73, 2004. (in Japanese)

16) Abe M, Suzuki Y, Hosoi $T$ et al. The pressure-pain threshold of mucosa under dentures. Part 1. the possible clinical application of the pressure-pain threshold meter. Tsurumi Shigaku 24:445-454, 1998. (in Japanese)

17) Gohdo Y, Fujisawa M. Determination of electromyogram biofeedback threshold for patients with clenching behavior. Prosthodont Res Pract 3: 46-54, 2004.

18) Muttray A, Kurten R, Jung D et al. Acute Effects of 200 ppm 1 1:1-trichloroethane on the Human EEG. Eur J Med Res 5:375-384, 2000.

19) Kim YK, Watanuki S. Characteristics of electroencephalographic responsesiInduced by a pleasant and an unpleasant odor. Appl Human Sci 22 : 285-291, 2003.

20) Uebaba K, Fenghao XU. Temperature-dependent physio-psychological changes by footbath : changes in electroencephalogram, cerebral circulation, R-R variability and comfort. J J A Phys M Baln Clim $67: 119-129,2004$. (in Japanese) 